\title{
La presencia de
}

Piaget en la Educación colombiana, 1960-2010

\section{// Piaget's Presence in Colombian Education, 1960-2010}

\section{Resumen}

El artículo realiza un recuento de diferentes formas de influencia que ha tenido el pensamiento piagetiano en la investigación educativa colombiana. Comienza con una serie de coincidencias entre la biografía de Piaget, la historia de la Educación colombiana en el siglo XX y algunas anécdotas de la vida del autor del artículo. Realiza luego un recuento de las experiencias educativas vinculadas con el Gimnasio Moderno en Bogotá, del trabajo de los profesores Carlo Federici Casa y Hernando Silva Mojica, y de los modelos educacionales integrados (MEI), hasta el planteamiento de la renovación curricular colombiana (1976-1994). En este último período, resume los debates entre los modelos educativos de inspiración conductista y constructivista. A partir de las principales críticas a las teorías piagetianas, el autor se reencuentra con los temas fundamentales de las teorías pedagógicas de los siglos XX y XXI.

\section{Abstract}

This paper develops a narrative about different ways of Piaget's thinking influence in Colombian educational research. That begins with a series of happenstances among Piaget's biography, the history of Colombian education in the 20th century, and some own anecdotes of the author of this paper. Then he recalls the educational experiences related to the school "Gimnasio Moderno" in Bogotá, works by professors Carlo Federici Casa and Hernando Silva Mojica, and the integrated educational models-IEM, up to proposals of Colombian curricular reform from 1976 to 1994. The last period summarizes debates between Behaviorism and Constructivism educational models. Starting from the main critical criticism to Piaget's theories, the author rediscovers seminal topics of pedagogical theories given during 20th and 21 st centuries.
}

\section{Palabras Clave}

Piaget, teorías pedagógicas, Educación colombiana, modelo conductista, constructivismo.

\section{Keywords}

Piaget, pedagogical theories, Colombian education, behavioral model, constructivism. 


\section{Introducción}

Comienzo con una serie de coincidencias entre la biografía de Piaget, la historia de la Educación colombiana en el siglo XX y las trivialidades de la vida de un apasionado por ambas.

Podríamos decir que las reformas y cambios en la Educación colombiana actual se inician con la Ley 39 de 1903 sobre la instrucción pública, ya hoy no muy apropiadamente llamada "Ley Uribe".

En ese año, Piaget entra a la escuela en Neuchâtel, Suiza, y a sus siete años empieza a interesarse por la mecánica, los pájaros, los fósiles y las conchas de moluscos, como nos dice en su autobiografía, y a escribir sus notas a lápiz porque a los niños no se les permitía escribir con tinta. Escribir y escribir fue lo que hizo en los primeros 80 años del siglo XX. Nos legó unos 80 libros, alrededor de 600 artículos y capítulos y más de 70.000 páginas manuscritas.

\section{Antecedentes}

\section{Médicos, psiquiatras y psicólogos en Francia, Suiza y Bélgica}

Después de la Segunda Guerra Mundial, en las carreras en Colombia se volvió muy frecuente estudiar textos en inglés y luego hacer estudios avanzados en los Estados Unidos. Pero antes de ella, de 1900 a 1939, la meca era Francia, Suiza y Bélgica.

De allá venían los textos más apreciados en todas las carreras, y allá iban los que querían saber más y más sobre arte, filosofía, medicina, derecho, ingeniería o literatura. Tengo una foto de mi padre, el Dr. Eduardo Vasco Gutiérrez, médico de la Universidad de Antioquia y primer psiquiatra infantil en Ilegar a Medellín, con Claparède, Bouvet y Piaget, codirectores del Instituto Rousseau de Ginebra a mediados de los años 30. Él, como muchos de los egresados de medicina en Colombia, había ido a estudiar en Ginebra, París y Bruselas lo que aquí nadie sabía.

Mucho más tarde, cuando regresé a Colombia en 1971con mi doctorado en matemáticas, mi padre me regaló un libro de Piaget sobre la probabilidad en los niños, publicación que había adquirido en un viaje a París en los años sesenta: La genèse de l'idée de hasard chez l'enfant (Paris: PUF, 1951), libro que también conservo con aquella valiosa foto. 
De los años 1925 a 1931 habían nacido los tres hijos de Piaget y Valentine Châtenay, Jacqueline, Lucienne y Laurent. Las observaciones sobre su desarrollo cognitivo dieron lugar a los tres libros más importantes de Piaget entre 1936 y 1945: El nacimiento de la inteligencia, La construcción de lo real en el niño y La formación del símbolo en el niño.

Para mí es muy simbólica la coincidencia de que en el año de mi nacimiento, 1937, Piaget publicó el libro La construction du réel chez l'enfant, traducido en Buenos Aires en 1965 en la Editorial Proteo. De ese libro tengo la reedición de 1976 en Ediciones Nueva Visión de Buenos Aires. La idea de construcción de lo real ha sido inspiradora para mí desde que me entusiasmé con la educación y la pedagogía en 1972 con el Profesor Carlo Federici Casa y luego con la maestría en Educación y Desarrollo Humano del Cinde, fundada por Marta Arango y Glen Nimnicht en el Colegio Cafam de Bogotá en 1976. Allí comencé con unas notas mimeografiadas para los estudiantes de la maestría- una reflexión que todavía continúo sobre las relaciones entre el conocimiento y la acción, los procesos y los sistemas, lo real y las realidades.

Pero volvamos al comienzo de la obra de Piaget, que inició en 1907 con la publicación de una nota sobre un gorrión albino cuando todavía estaba en el colegio, y con una serie de observaciones sobre moluscos que comenzó en 1911 y hasta incluye quién hubiera pensado en esa coin- cidencia- algunos estudios de los moluscos de Colombia'.

A pesar de que su obra epistemológica y psicológica no había comenzado aún en esos años iniciales del siglo $X X$, ya encontramos allí una primera puerta hacia la entrada de Piaget a la educación colombiana.

\section{Decroly en el Gimnasio Moderno}

Como tantos otros jóvenes ávidos de saber, Agustín Nieto Caballero viajó a Europa en 1910. Allá conoció a Ovide Decroly y a María Montessori y regresó a Bogotá en 1913, entusiasmado con las ideas Ilamadas entonces la "Escuela Nueva". En 1914 fundó el Gimnasio Moderno². Años más tarde, 1925, Decroly visitó personalmente el Gimnasio Moderno, en donde se sorprendió de la riqueza y creatividad de la adaptación de sus ideas por el Dr. Nieto Caballero.

En ese momento, Piaget apenas empezaba su vida académica, pues en 1921 había llegado al Instituto Rousseau. En 1924 -antes de que nacieran sus hijos- salieron sus dos primeros libros: El lenguaje y el pensamiento en el niño y El juicio y el razonamiento en el niño, los únicos que pudo conocer Vygotsky y que llevarían a la controversia entre piagetianos y vygotskianos.

\footnotetext{
Ver su autobiografía en Internet, en el capítulo I: 1896-1914 en la página de la Fundación Jean Piaget, en el URL http//www/fondationjeanpiaget.ch

2 En la historia ilustrada en la página de Internet del Gimnasio Moderno hay simbólicas fotografías de don Agustín con Decroly, con María Montessori y con Piaget, lamentablemente sin fechas.
} 
Las ideas iniciales de la pedagogía de Nieto Caballero fueron pues independientes de Piaget, e inspiradas más que todo en las pedagogías montessoriana y decroliana. El primer lazo directo con Piaget parece haber sido la traducción y adaptación de los juegos piagetianos de La Maison des Pétits de Ginebra con los de Decroly, los de Montessori y los de Bradley³.

De ahí en adelante, las ideas de Piaget, de Dewey y de Giner de los Ríos se mezclaron con las de Decroly y Montessori en la mente creativa de don Agustín, quien inspiró a muchos educadores y educadoras colombianos no solo en el Gimnasio Moderno y luego en el Gimnasio Femenino, sino también en sus años en la Dirección General de Educación y la Inspección de Educación Primaria y Normalista durante los gobiernos de Olaya Herrera y Alfonso López Pumarejo (1932-1936).

El Gimnasio Moderno y el Femenino siguieron acogiendo y adaptando ideas piagetianas después de la Segunda Guerra Mundial, pues en 1945 volvió don Agustín como Rector, hasta el año 1975 en que murió.

Infortunadamente, como lo veremos en seguida, desde los años 70 se dio entre los directivos de la educación privada en Bogotá una identificación de las ideas piagetianas con las propuestas de los Métodos o Modelos Educacionales Integrados, MEI de Carlo Federici, Germán Zabala y Hernando Silva Mojica, lo que llevó a considerar esas ideas como sospechosas de marxismo y a reducir en muchos colegios la experimentación y los cambios a las propuestas que Ilegaban desde la Tecnología Educativa y el Análisis Experimental de la Conducta ${ }^{4}$.

\section{Carlo Federici Casa y Hernando Silva Mojica}

La lógica matemática de Russell y Whitehead empezó a filtrarse en los cursos de lógica aristotélica de la Universidad Javeriana apenas 50 años después de los Principia Mathematica en 1960. El P. Jesús Sáenz, mi profesor de lógica, y el P. Jaime Vélez, mi profesor de historia de la filosofía, invitaron a Carlo Federici a

3 Ver Quiceno, H. (2007). Agustín Nieto Caballero (1889-1975). En S. CastroGómez y otros (Eds.), Pensamiento colombiano del siglo XX (vol. 1, pp. 15-31). Bogotá: Pontificia Universidad Javeriana-Instituto Pensar

4 Peralta, María Victoria (2008). En el centenario de l'École Decroly 1907-2007: La pedagogía decroliana en Latinoamérica y la visita del Dr. Decroly a Colombia (1925). Santiago de Chile: Universidad Central.

Decroly, Ovide (1932). El doctor Decroly en Colombia. Inspección Nacional de Educación Primaria y Normalista. Bogotá: Imprenta Nacional. 
trabajar con nosotros, los estudiantes de la licenciatura en filosofía y letras, en la formalización de las conectivas lógicas del cálculo proposicional. Federici según las ideas de Piaget sobre la génesis del número en el niño, hizo también unas sesiones demostrativas sobre el aprendizaje de la aritmética por alumnos de primaria.

Federici tenía en francés las obras de Piaget sobre las 16 conectivas lógicas binarias y las 256 ternarias: Traité de logique: essai de logistique opératoire, (Paris: Dunod, 1949) y Essai sur les tranformations des opérations logiques: les 256 opérations ternaires de la logique bivalente des propositons (Paris: PUF, 1952).

Sin embargo, Federici consideraba que era mucho mejor su propio tratamiento de las conectivas con la notación que había desarrollado en Italia en los años 30 en la Universidad de Génova con Alessandro Padoa, discípulo de Peano. Piaget ya estaba allí en ese Bogotá de los años 60, con adopción y adaptación, admiración y crítica.

En 1961 recibí clases de psicología del P. Hernando Silva Mojica, quien había llegado recientemente de Roma con un doctorado en filosofía. Su tesis doctoral había tomado de la epistemología de Piaget algunas críticas a las teorías del conocimiento clásicas, tomistas, cartesianas y empiristas. Sus estudios sobre Piaget lo habían iniciado en el camino de reformular el realismo tomista y la teoría escolástica de los conceptos por una psicología genética de construcción activa del conocimiento por parte de los niños y jóvenes.

Las posiciones innovadoras de los jóvenes profesores tienen riesgos y despiertan muchas reacciones. Desde su llegada de Roma a Bogotá, el P. Silva encontró críticos y contradictores aun entre los mismos jesuitas; pero también encontró interlocutores entusiastas en Carlo Federici y Germán Zavala; a quienes pronto se les unieron Beatriz Farías, Clara Helena Sánchez y Fabiola Rodríguez, y que más adelante conformaron, junto con otras alumnas de Federici el que podríamos Ilamar "el primer grupo Federici". Las discusiones sobre Piaget y su aplicación a la educación siguieron entre ellos con creciente número de participantes durante toda la década de los 60 .

\section{Los Modelos Educacionales Integrados o Método de Educación Integrada MEl y el caso Marymount}

Como Agustín Nieto en 1910 o Eduardo Vasco en 1930, en 1965 va Germán Zabala a París, donde estudió las corrientes neomarxistas más recientes y entró en contacto con las matemáticas bourbakistas, de las cuales tomó Piaget muchas de sus ideas sobre las estructuras, la lógica y los conjuntos. A su regreso de París, Zabala se adhiere al Frente Unido del padre Camilo Torres Restrepo. Este, después de sus estudios en Lovaina, 
había regresado a Bogotá en 1959. Inició con Orlando Fals Borda la Facultad de Sociología de la Universidad Nacional, de la cual tuvo que salir en 1962.

Con su proclama a los cristianos en ese año sobre la necesidad de estar del lado de los marxistas en la lucha por una nueva sociedad, cambió el panorama de la Iglesia Católica colombiana y se produjo una profunda división entre los católicos.

El Frente Unido se rompe ante la represión oficial. Camilo Torres se va a la guerrilla, y muere en 1966 en su primera acción armada en Patio Cemento, Santander.

Germán Zabala continúa con sus ideas educativas con grupos marxistas en el trabajo que él llamaba "la universidad militante", del que saldría la nueva Universidad INCCA de Jaime Quijano Caballero, y con grupos cristianos en la educación primaria y secundaria ${ }^{5}$.

La Iglesia Católica latinoamericana estaba cambiando muy lentamente después del Concilio Vaticano Segundo (1962-1965), pero en 1968, en la Conferencia Episcopal Latinoamericana Celam de Medellín, los obispos se comprometieron públicamente con una radical opción por los pobres. En toda la América Latina se generaron discursos teológicos revolucionarios en el intento de síntesis de marxismo y cristianismo que se empezó a llamar "Teología de la Liberación".

Surgió en Colombia el grupo "Golconda" de sacerdotes de izquierda, al que estuvieron muy cercanos los hermanos Germán y Manuel Zabala. Las ideas de Freire sobre la educación liberadora, publicadas en 1967 en el libro Educación como práctica de la libertad y sus críticas a la "educación bancaria" se empezaron a expandir en toda la educación formal pública y privada y en la educación popular.

Germán Zabala, el P. Hernando Silva Mojica y otros sacerdotes y religiosas comprometidas con el cambio social, diseñaron con Carlo Federici una serie de modelos innovadores de educación crítica y política muy eclécticos, basados en ideas marxistas, freireanas y piagetianas. Se llamaron "Modelos Educacionales Integrados MEI", sigla también interpretada por algunos como "Método de Educación Integrada". 
Estos métodos y modelos se empezaron a experimentar en algunos colegios parroquiales en Colombia y Venezuela, y en forma más sistemática en un colegio del Barrio Galán de Bogotá, dirigido por sacerdotes consolatos, en donde enseñaban unas pocas religiosas del Sagrado Corazón de María. Algunas de estas religiosas ensayaron estas unidades integradas MEI con sus alumnas de clase alta en el Colegio Marymount al norte de Bogotá, con gran alarma de las familias de las alumnas, de sacerdotes y religiosas opuestos a cualquier contaminación del cristianismo por el marxismo. Pronto empezaron a llegar denuncias al Arzobispo de Bogotá, al Nuncio de su Santidad y a las superioras de la comunidad del Sagrado Corazón de María en Roma y Estados Unidos.

Un incidente simbólico en un acto público del Colegio Marymount a comienzos de 1969 fue interpretado por los padres y madres de familia como la "prueba reina" de que el MEI era un método de indoctrinación marxista. La reacción fue inmediata: el Cardenal Aníbal Muñoz Duque expulsó a todas las religiosas del Marymount de la arquidiócesis de Bogotá y la comunidad tuvo que entregar el colegio a la asociación de padres de familia.

Así, los experimentos MEI terminaron abruptamente, y las ideas piagetianas y freireanas se asociaron en el imaginario de las clases dirigentes colombianas con las marxistas. Se recordó que la Santa Sede le había prohibido al P. Silva la enseñanza de la filosofía en la Universidad Javeriana por defender tesis de Darwin y de Piaget contrarias a la psicología escolástica. Se revivió la leyenda negra de que Federici había venido a Bogotá con Fidel Castro en 1948 a organizar la sublevación del 9 de abril, y aun el alto gobierno intentó deportar a Federici en 1970.

Algunas religiosas del Marymount se fueron a Medellín y Barranquilla, y otras regresaron a Estados Unidos. Carlo Federici, Germán Zabala y Hernando Silva se restringieron prudentemente a investigaciones $y$ publicaciones sobre la Educación, las Matemáticas y la Lógica con las ideas de Bourbaki y de Piaget.

Yo regresé de Alemania en octubre de 1971, e inmediatamente retomé contacto con mi antiguo profesor de los años 60 y 61, quien me había dirigido la tesis de licenciatura en Filosofía sobre la Epistemología del espacio y el tiempo en la relatividad especial einsteiniana.

Tuvimos innumerables reuniones en su casa, a las que iban con frecuencia muchos alumnos y alumnas suyos de la Universidad Nacional, algunos jesuitas, Germán Zabala y su hijo Vladimir, y otros intelectuales colombianos, españoles e italianos.

De ese tiempo datan los libros del P. Silva con Federici y sus discípulas sobre los números enteros publicados por la Universidad Javeriana y las guías para la enseñanza de la Matemática en bachillerato de Federici con Clara Helena Sánchez 
y Fabiola Rodríguez de Loboguerrero, publicados por el Cias, como se Ilamaba entonces el Centro de Investigación y Educación Popular Cinep, en 1972.

En ese mismo año 1972, el CIAS publicó como número 10 de la Colección Monografías y Documentos, el libro "Educación para el cambio", del P. Hernando Silva. En ese libro se retomaban ideas de la Psicología, como la conceptualización de la adaptación como asimilación y acomodación de Piaget; del Psicoanálisis, especialmente de Erich Fromm, Herbert Marcuse y otros autores sospechosos de Marxismo, que el Padre Silva matizaba con abundantes referencias a documentos oficiales del episcopado colombiano y latinoamericano, sobre la no-violencia de Ghandi y con extensas citas de Martin Luther King.

Federici se dedicó a la Educación y la Pedagogía. En la década de los 70 dirige el departamento de Ciencias de la Educación y después, el departamento de Pedagogía de la Universidad Nacional. En ratos libres, empezó a colaborar como asesor del Instituto de Ciencias, filial del Instituto Colombiano de Pedagogía Icolpe, adscrito a la Universidad Pedagógica Nacional. Me invitó a trabajar con él, con Ramón Espinosa Luppi, los esposos Mary Falk y Ricardo Losada y otros colegas de la Nacional y de otras universidades de Bogotá. Allí empezamos a investigar y experimentar la posibilidad de enseñar las Matemáticas modernas, en especial la teoría de conjuntos y la lógica, con las ideas de Bourbaki y de Piaget, de 1972 a 1974. De esas discusiones y experimentaciones salió mi primera ponencia sobre Educación Matemática, que presenté en la Cuarta Conferencia Internacional de Educación Matemática en Caracas en 1975 y fue publicada al año siguiente por la Unesco en Uruguay ${ }^{6}$.

\section{Conductismo vs. estructuralismo en Psicología}

Esa misma década de los setenta vivió una guerra sorda entre la psicología de Watson y Skinner: el Análisis Experimental de la Conducta, que llegó a considerarse como la única Psicología científica y acaparó las revistas de Psicología, los congresos y los fondos de investigación.

6 Vasco, C. E., Falk de Losada, M., Charris Castañeda, J. y Losada Márquez, R. (1976). Consideraciones sobre la enseñanza de la matemática en el ciclo diversificado colombiano. En: Unesco-Ciaem, Educación Matemática en las Américas-IV. Montevideo, pp. 97-114. 
En nuestras universidades llegó a considerarse que los pocos psicólogos estructuralistas, piagetianos o de la Gestalt, los fenomenólogos y los seguidores de las distintas escuelas psicoanalíticas eran apenas mitómanos que engañaban a sus estudiantes con discursos oscuros y pseudocientíficos. Era imposible elegir un director de departamento que no fuera conductista, y los alumnos de ambos bandos dejaban grafitos en el tablero para insultar a los de la siguiente clase.

En 1973, el rector de la Universidad Javeriana, el P. Alfonso Borrero, y el rector de la Universidad de los Andes, el Dr. Jorge Ortiz Méndez, llegaron al acuerdo de concentrar a los más ortodoxos conductistas en los Andes y a los más aguerridos estructuralistas en la Javeriana. En 1973, en la Facultad de Psicología de la Javeriana se creó el departamento de Psicología Educacional, en donde los estructuralistas pudieron respirar tranquilos e iniciar exploraciones e investigaciones piagetianas. Recuerdo al menos tres decanos de esta inclinación: Graciela Aldana de Conde, Arnoldo Aristizábal y la entonces estudiante Ángela María Robledo. Con Carlo Federici, Hernán Escobedo, Jorge Castaño, Juan Carlos Negret y muchos otros, trabajamos en consolidar una Psicología educativa piagetiana en la Universidad Javeriana.

En la Universidad Nacional no fue fácil resolver los conflictos en- tre conductistas y estructuralistas. Pero en ese entonces, se dieron dos "conversiones" cruciales para el desarrollo de las ideas de Piaget en la educación colombiana: las de Félix Bustos Cobos y Hernán Escobedo David. De serios y bien documentados conductistas, a través de sus lecturas e investigaciones descubrieron la Psicología genética y las ideas epistemológicas de Piaget y las abrazaron y promovieron activamente.

Félix Bustos fue nombrado como director del departamento de Psicología de la Nacional, traído en comisión del MEN para conciliar entre conductistas y estructuralistas. Invitados por él, Federici y yo tuvimos una serie de reuniones y conferencias sobre Piaget en la Universidad Nacional, y Félix Bustos logró abrir un nicho permanente para la investigación y la docencia de la Psicología genética.

Fue clave en el ocaso de la hegemonía conductista y en el auge del constructivismo la difusión de la obra de John H. Flavell: The developmental psychology of Jean Piaget (Princeton, NJ: van Nostrand, 1963), traducida al castellano como "La Psicología evolutiva de Jean Piaget" (Buenos Aires: Paidós, 1971) y reeditada y reimpresa muchas veces. Yo tengo la $5^{\text {a }}$ edición en castellano, casualmente también de 1978, el año en que empecé a asesorar al Ministerio de Educación para la elaboración de los programas de matemáticas de la Renovación Currricular. 


\section{La renovación curricular de 1976 a 1994}

\section{La tecnología educativa y el diseño instruccional}

En el gobierno de López Michelsen (1974-1978), después de un primer período en el que el entonces Ministro de Educación, Hernando Durán Dussán, logró ampliar drásticamente la cobertura con el artificio de decretar la división del tiempo escolar en tres jornadas, un grupo de educadoras lideradas por Pilar Santamaría de Reyes y Clara Franco de Machado, empezaron a preocuparse por las consecuencias negativas de dichas medidas. Introdujeron en el país el entonces nuevo discurso de la calidad y del mejoramiento cualitativo de la Educación en 1975. En 1976 habían logrado lo que parecía imposible: la creación de la Dirección General de Capitación y Perfeccionamiento Docente, Currículo y Medios Educativos, separada de la Inspección Nacional del Ministerio de Educación. Pilar Santamaría fue la primera directora general, y Clara Franco la primera jefe de la división de currículo.

Con asesores de la OEA, la Unesco y los Cuerpos de Paz, se inició prontamente, aun antes del traslado a la sede del norte de Bogotá, el diseño de nuevos programas curriculares de primero a tercero de primaria, para remplazar los de 1963. En ese momento, no se dudaba de que ese diseño de los nuevos programas debería ser con la tecnología educativa en boga, basada en el Análisis Experimental de la Conducta. Las personas que se habían preparado en Stanford o Tallahasee, en Sao Paulo o en Campinas, tenían listo el arsenal de modelos y métodos para diseñar rápidamente los programas de cada área.

Profesionales técnicos, directivos y asesores recibían capacitación en tecnología educativa y diseño instruccional (TEYDI). Se crearon en la Dirección General grupos de Matemáticas, de Ciencias Naturales, de Ciencias Sociales, de Lenguaje (Español y Literatura) y de las Ilamadas "educaciones": Física, Estética, Tecnológica, Religiosa, Moral y Ética.

No existía todavía una crítica académica externa que pudiera hacer cuestionamientos fuertes al Análisis Experimental de la Conducta y a la tecnología educativa de corte conductista. No había todavía un movimiento pedagógico fuerte en el magisterio; solo se concretó hacia 1984, y apenas empezaba en Medellín el Grupo de Historia de las Prácticas Pedagógicas dirigido por Olga Lucía Zuluaga. 
Empezaba apenas la expansión de las ideas de Freire, y estaban Ilegando todavía muy esporádicamente las críticas desde la sociología, como las de Bourdieu y Passeron al enfoque durkheimiano de la Educación. Basil Bernstein no se conocía, y solo los psicólogos gestaltistas y piagetianos cuestionaban críticamente la hegemonía de la Psicología conductista.

La crítica a la tecnología educativa y al diseño instruccional no vino pues de fuera, sino que se inició en forma interna en el MEN con la llegada de Félix Bustos y Campo Elías Burgos a la nueva dirección general, con el nombramiento por parte de la Universidad Nacional de Carlo Federici como asesor de los grupos de Matemáticas y Ciencias Naturales de 1976 a 1977 y de Daniel Herrera como asesor del grupo de Sociales.

En 1978, el Dr. Federici se jubiló y la Universidad Nacional me nombró para sucederlo como asesor del grupo de Matemáticas del Ministerio de Educación.

Con los colegas mencionados antes y con algunos profesionales técnicos de los distintos grupos de trabajo, pudimos ubicar el discurso piagetiano contra el conductista en tres frentes: la Epistemología, la Psicología y la Pedagogía. El problema mayor se dio en la Psicología, especialmente en el tema del aprendizaje.

Aunque todavía faltaban tres años para el simposio del Planetario Distrital de 1981 y otros tres para la adopción de los programas curricu- lares por el Decreto 1002 de 1984, los debates internos en el Ministerio pueden seguirse de cerca si se hace un análisis de texto cuidadoso del libro Fundamentos generales del currículo (Bogotá: MEN-DGC, 1984), llamado "el libro azul", que se gestó de 1980 a 1984 y con el cual se iniciaban los cursos de capacitación para la renovación curricular. En ese delgado folleto de 52 páginas pueden notarse claramente los tres frentes del debate interno entre piagetianos y conductistas.

En los fundamentos epistemológicos (pp. 27-31), como los conductistas, no creían en ese discurso ni pensaban necesitarlo, pudimos silenciar el positivismo e introducir la Epistemología habermasiana y la piagetiana. Tres líneas de la p. 28 son suficientes:

Particular importancia tiene la epistemología genética de Jean Piaget en tanto que es una de las más fecundas en lo que se refiere a la comprensión de los procesos de desarrollo del niño.

En los fundamentos psicológicos (pp. 37-46) la negociación fue dura. Respecto a la inteligencia, pudimos utilizar las ideas piagetianas del animismo, el realismo ingenuo, la que llamamos "pensar configurativo" y el egocentrismo infantil (pp. 39-40) sin objeciones de los colegas.

Pero, como era de esperarse, al tratar sobre el aprendizaje no fue posible llegar a acuerdos. Se optó por poner en paralelo los cuatro mo- 
delos en conflicto, para que los maestros escogieran el que más les convenciera: el modelo de Piaget, el modelo de Skinner, el de Bandura y el de Gagné (pp. 42-44). Les seguían las respectivas consecuencias para el currículo, también por separado (pp. 4446), en las que logramos evitar descalificaciones mutuas y juicios de valor. Al encontrar divididos a los conductistas, lo más que logramos fue ubicar el modelo piagetiano en primer lugar.

En los fundamentos pedagógicos (pp. 47-52) no hubo necesidad de discusiones. Para los conductistas, la pedagogía era un discurso filosófico sobre la educación sin consecuencias concretas en los programas curriculares de las áreas. El tecnólogo educativo recibía del Ministerio los fines de la educación y los contenidos y objetivos generales de cada área, y apenas allí empezaba su trabajo. Los aspectos didácticos podían resolverse con un buen diseño instruccional, con objetivos específicos bien desmenuzados y ordenados, con actividades detalladas, recursos e indicadores de evaluación. Sobraban los discursos pedagógicos y didácticos.

Por eso, pudimos redactar a nuestro modo el aparte sobre la pedagogía activa y mencionar sin ambages a Dewey, Claparède, Binet, Ferrière, Kerschensteiner y Piaget (p. 47). En la última página de consecuencias para el currículo (p. 52), terminamos con una muy tensa mezcla de pedagogía activa y tecnología educativa, que a más de 25 años de distancia se lee con una no muy tensa mezcla de ceño fruncido y sonrisa benévola.

Pero desde 1978, el enfoque piagetiano había entrado de lleno en el grupo de Matemáticas. A mi llegada en marzo de 1978 a remplazar a Carlo Federici, estaba allí Cecilia Casasbuenas, recién llegada de París, en donde conoció a Régine Douady y la naciente didáctica francesa de los piagetianos Gérard Verganud y Guy Brousseau.

Luego Ilegaron Teresa León, Celia Castiblanco, Carmen Lucila Osorno, Gabriel Gutiérrez, Orlando Múnera y Virginia Cifuentes, con quienes trabajamos los enfoques piagetianos en la construcción del número y el espacio.

Las ideas constructivistas piagetianas habían sido ya sembradas por Carlo Federici en el grupo de Ciencias Naturales, con Edith Figueredo de Urrego, Luis Eduardo García, Doris Amanda Espitia, Henry Realpe, Jorge Arias, Gladys Prada y otros. Continué asesorándolos de vez en cuando, pero con ellos la didáctica constructivista no necesitaba más apoyo. 
En el grupo de Ciencias Sociales, compuesto inicialmente por Antonio Rivera, Nerehey Ortega y César Ledesma, había más bien un rechazo a la tecnología educativa por las posiciones antiescuela de algunos grupos del magisterio, a los que todavía no habían Ilegado las propuestas positivas derivadas de la Psicología vygotskiana. Pero pronto llegaron Rosario Jaramillo y Pilar Vargas, Mercy Abreu de Armengol y Nedgidia Fernández, y luego Ángela Bermúdez y Ana Victoria Navarro, con ideas epistemológicas antipositivistas y convicciones pedagógicas de la escuela activa y el constructivismo.

Por esa misma época, se extendió a otras cohortes abiertas la maestría del Cinde mencionada al comienzo, que fue inicialmente de admisión cerrada para los docentes del Colegio Cafam. Allí entró en 1977 Eloísa Vasco Montoya a coordinar las maestría y a contribuir como profesora a la maestría de Cafam y a las abiertas, en las cuales se trabajó con posiciones constructivistas. Ella misma empezó un doctorado con la Universidad de Nova y el Cinde, y desarrolló un concienzudo proyecto sobre desarrollo del pensamiento formal en los estudiantes del Colegio Cafam. Esa fue su tesis doctoral, terminada en 1981, dirigida por mí y por Carlos Rojas, que fue seguida por un proyecto de Colciencias en distintos colegios de Bogotá y Cundinamarca. Fue el inicio de una serie de investigaciones sobre el pensamiento formal con marco teórico piagetiano en varias regiones del país.

\section{El simposio del Planetario Distrital en 1981}

En ese mismo año 1981, el Ministerio de Educación decidió dar por terminada la experimentación curricular en los cinco grados de primaria; pero antes de expedir los programas curriculares por decreto, envió para su evaluación a todas las facultades de Educación y a las universidades Nacional y de los Andes las propuestas de los programas curriculares de Matemáticas y de Ciencias Naturales. El Ministerio citó a las universidades a un simposio nacional sobre los nuevos programas curriculares en el Planetario Distrital de Bogotá.

Ninguna facultad de educación asumió el reto de evaluar esos programas, pero afortunadamente la Universidad de los Andes y la Nacional sí encargaron grupos de profesores para ese trabajo. El grupo de Matemáticas y el de Física de los Andes trajeron juiciosas críticas y comentarios para mejorar esos dos programas, y así lo hizo también el grupo de la Universidad Nacional, con Mary Falk de Losada, Myriam Acevedo y Crescencio Huertas, con el programa de Matemáticas.

Pero el grupo de la Universidad Nacional encargado por la Facultad de Ciencias de evaluar los programas de Ciencias Naturales no se limitó a estudiarlo. Era precisamente el segundo grupo Federici, con Antanas Mockus, Carlos Augusto Hernández, José Granés, Berenice Guerrero, Jorge Charum, Luz Marina Caycedo y otros más. 
Los colegas del grupo Federici criticaron finamente el programa de Ciencias Naturales, pero se extendieron a una amplia y aguda crítica a la idea misma de producir programas curriculares con la tecnología educativa conductista, que denunciaron como degradación positivista de la didáctica; al desmenuzamiento de los contenidos en objetivos específicos, que tildaron de "taylorización de la Educación" a la instrumentalización del maestro por el diseño instruccional que pretendía desarrollar currículos "a prueba de maestros", lo que consideraron una ofensa a la dignidad del maestro como intelectual orgánico y trabajador de la cultura.

Fue un golpe muy duro, más bien una azotaina contundente, que le dolió a algunos y pareció injusta a muchos. Pero a quienes habíamos resistido la presión conductista y trabajábamos dentro del Ministerio con los enfoques piagetianos, tales críticas nos dieron la razón. El Dr. Miguel Ramón, director general de capacitación, currículo y medios, detuvo por tres años la expedición de los programas y exigió una revisión completa de los marcos teóricos y los diseños grado por grado que pudiera dar la mayor seriedad a la propuesta. Tres años de duro trabajo interno transcurrieron antes de que la Ministra Doris Eder de Zambrano expidiera los programas de la renovación curricular por el Decreto 1002 de 1984.

En ese tiempo de 1981 a 1984 se configuró el "libro azul" del que hablamos arriba, y el "libro amarillo" con los marcos generales de los programas curriculares, publicado también en 1984 por el MEN y la DGC, además de los cinco tomos de programas grado por grado.

\section{El auge del constructivismo en Educación}

En los diez años desde la expedición de los programas del Decreto 1002 de 1984, hasta su derogación por la Ley General de Educación en 1994, podemos hablar de un auge del constructivismo en la educación, a la vez que de un auge del Movimiento Pedagógico del magisterio. Esta segunda corriente ascendente llevó en diez años a suprimir de un tajo la renovación curricular, sin siquiera haber comenzado todavía en la educación secundaria.

Sigamos algunas huellas del discurso piagetiano y el constructivismo genético a través de algunos de mis trabajos con otros colegas en esas dos décadas de 1980 a 2000.

El simposio sobre psicología unificada, con Hernán Escobedo y otros colegas en 1984, invitó a psicólogos y educadores, de muy 
amplio espectro, a defender sus ideas sin atacar a los demás ${ }^{7}$.

Mi trabajo de ascenso a profesor titular, un extenso libro sobre el constructivismo genético terminado en Harvard en 1985, fue entregado por la Facultad de Ciencias con orden de publicación a la Imprenta de la Universidad Nacional. Por razones burocráticas nunca se publicó. Solo se logró la reproducción de algunos capítulos en los libros del Ministerio de Educación sobre el enfoque didáctico del programa de Matemáticas del Decreto 1002 de 1984, que se utilizaron ampliamente en los cursos de capacitación y perfeccionamiento docente ${ }^{8}$.

Mencionaré también el seminario de procesos de pensamiento que organizó Félix Bustos en el MEN, del que salió el libro de memorias del seminario publicado en 1991, OEAMEN-DGC (1991). Desarrollo de procesos de pensamiento-Memorias [Serie "Pedagogía y Currículo", n. 5]. Santafé de Bogotá, MEN-DGC.

7 ICFES (1984). Simposio: ¿Es Posible una Psicología Unificada? [Serie Memorias de Eventos Científicos Colombianos, n. 22]. Bogotá: ICFES/ Guadalupe.

8 Vasco, C. E. (1984). Un Nuevo Enfoque para la Didáctica de las Matemáticas. Volumen I. Bogotá: Ministerio de Educación Nacional. Segunda edición: 1994. Vasco, C. E. (1988). Un Nuevo Enfoque para la Didáctica de las Matemáticas. Volumen II. Bogotá: Ministerio de Educación Nacional. (Segunda edición: 1994).

La Editorial Norma hizo una edición parcial gratuita del primer volumen: Vasco, C. E. (1986). El Enfoque de Sistemas en la Enseñanza de la Matemática. Bogotá: Editorial Norma. Un tercer volumen que editó Martha Osorno en 1994 nunca se imprimió y solo circuló en fragmentos fotocopiados.
En el apéndice I doy las referencias a una serie de artículos míos que muestran en sus títulos algunas trayectorias de difusión y discusión del constructivismo piagetiano en esas dos décadas de 1980 a 2000.

Una lista semejante podría hacerse sobre las publicaciones de Félix Bustos Cobos y sobre las traducciones de artículos y capítulos pertinentes que hizo durante esos años.

Podría recordar también algunos seminarios sobre Piaget en la Universidad Javeriana, especialmente con Martha Vargas de Avella y Elba Martínez de Dueri en Educación, con muchas proyecciones a cursos con maestros y maestras de todo el país.

Recuerdo también algunos seminarios con los colegas del departamento de Física en la Javeriana, Dino Segura, Alfonso Suárez, María Barrera de Aragón y Jorge Zamora.

Dino Segura siguió su propia línea piagetiana, enriquecida después con sus ideas educativas que podrían clasificarse como críticas al constructivismo o como radicalizaciones del constructivismo. La producción académica, didáctica y sobre todo, el impacto en los docentes y estudiantes de la Escuela Pedagógica Experimental EPE no son cuantificables.

En esta vertiente del constructivismo, en el trabajo por proyectos integrados es especialmente notable la larga trayectoria de la EPE, inicialmente con Adela Molina y luego con muchos otros docentes en la teorización, desarrollo e implementación de las Actividades Totalidad Abiertas ATA's. 
Los otros colegas del departamento de Física en la Javeriana ya mencionados, Alfonso Suárez, María Barrera de Aragón y Jorge Zamora, y luego María Victoria Quijano de Matemáticas, inicialmente discutieron contra la Epistemología piagetiana desde la bachelardiana. Los diálogos y seminarios sobre estos dos autores llevaron a que nos constituyéramos en grupo de investigación: el Grupo de Epistemología de la Facultad de Ciencias de la Javeriana. En ese grupo trabajamos nuestra propia versión del constructivismo desde el punto de vista histórico-epistemológico. Con ellos cuatro comenzamos una especialización en docencia de las Ciencias Naturales y las Matemáticas desde el punto de vista de la construcción del conocimiento.

En la especialización contribuyeron luego Nubia Perdomo de Parra, Julio Latorre, Raúl González, María Haydée Becerra y otros egresados de la misma especialización. Este programa tuvo muchas promociones y publicaciones. En el apéndice II menciono las más importantes.

\section{Otras proyecciones de las ideas piagetianas en Colombia}

No puedo dejar de mencionar los libros de Rómulo Gallego Badillo sobre el constructivismo y los distintos libros con Royman Pérez y otros colaboradores y artículos producidos por él sobre este tema durante esos mismos 20 años, especialmente influyentes en la enseñanza de la Química y de las Ciencias Naturales en general.

Debo dar tributo al grupo "Dimensión Educativa" con Germán Mariño, Mario Peresson, Lola Cendales y muchos otros colegas, quienes en todas las áreas curriculares en la educación formal y en la educación popular, articularon muy bien las ideas de Freire y las de Piaget.

Fue larga y fecunda la trayectoria de los cursos de capacitación de los Centros Experimentales Piloto CEP del Ministerio de Educación Nacional en todo el país desde 1976 a 1994. En particular, en Medellín, con Irma Hurtado como organizadora y maestra, las ideas piagetianas en la didáctica de las Matemáticas tuvieron como líder a Orlando Mesa Betancur, quien fue el primero que se atrevió a combinar ideas vygotskianas con las piagetianas y con otras de su propia cosecha en los cursos de capacitación sobre enseñanza de la Aritmética elemental.

En Cali, el grupo GEM con Gloria Castrillón, Jorge Arce y otros colegas, extendieron por todo el Departamento del Valle estas ideas piagetianas en la geometría. En el CEP de Tunja tuvo gran impacto en toda la región cundiboyacense con Rosario Nájar como líder y con los aportes de Miguel Díaz, Publio Suárez, Germán Torres y otros colegas 
de la Asociación Boyacense de Matemáticas y Física.

Varios grupos de lengua materna en todo el país trabajaron y siguen trabajando la didáctica de la lectura desde el enfoque piagetiano de Emilia Ferreiro, Anna Teberosky y Ana María Kaufmann. En particular, menciono a Juan Carlos Negret y el Grupo Herramientas y Gestión en Bogotá y en Medellín, a Octavio Henao y su grupo.

Por supuesto que, además de la didáctica de las Matemáticas, fue la didáctica de las Ciencias Naturales la que más extendió las ideas piagetianas por todo el mundo y, a través de los grandes maestros, también a Colombia. La didáctica constructivista la iniciaron en los Estados Unidos, MacCloskey, Clements, Caramazza y McDermott; en Francia, Louise Viennot; en Inglaterra, Rosalind Driver; en España, César Coll, Juan Ignacio Pozo, Mario Carretero, Eduardo Martí y Carmen Gómez-Granell. Sus discípulos y lectores asiduos en Colombia son innumerables. Aunque dar unos pocos nombres sea inequitativo con tantos colegas entusiastas, quiero recordar ahora solo a algunos con quienes trabajé directamente en la enseñanza de las Ciencias Naturales y las Matemáticas: el profesor Alfonso Claret Zambrano en Cali y el profesor Miguel Monsalve en Medellín.

Sin embargo, como valoración global de estas dos décadas de auge del constructivismo piagetiano en la educación colombiana, tengo que ser realista. Podríamos decir que tuvimos demasiado éxito en la adopción de los discursos constructivistas por parte de los y las docentes de todas estas áreas curriculares. En las reuniones de los años 90 podríamos fácilmente oír frases como "Ahora todos somos constructivistas". Cuanto de ese discurso se tradujo en cambios en el aula, en la evaluación y en la vida extraescolar está todavía por verse. Es posible que casi todos los profesores y profesoras nos hubiéramos convertido al constructivismo, al menos de palabra, pero me temo que la mayoría de nuestros estudiantes no se dieron cuenta del cambio.

\section{Las críticas a Piaget \\ La crítica interna entre los discípulos de Piaget}

En esos mismos veinte años ocurrieron múltiples procesos de crítica, extensión y rechazo a las ideas de Piaget. Con razón muchos psicólogos y epistemólogos piagetianos sostuvieron que sus entusiastas seguidores habíamos extrapolado inválidamente ideas epistemológicas y psicológicas a la didáctica, cuando no eran apropiadas para el aula o al menos no habían sido refinadas lo suficientemente para serlo.

Los pospiagetianos, como Bärbel Inhelder y sus colegas Pierre Gréco, Christianne Gillieron, Montserrat Moreno y Genoveva Sastre, criticaron los métodos de entrevista piage- 
tianos y los intentos de reducirlos a estructuras mentales universales. En Cali, Mariela Orozco y Rebeca Puche lideraron esta línea, trajeron al país a sus mejores exponentes y publicaron los primeros libros y artículos sobre la corriente pospiagetiana.

Los neopiagetianos no criticaron directamente a Piaget, sino que trataron de insertar sus ideas en las nuevas vertientes norteamericanas de la Psicología Cognitiva, la Inteligencia Artificial y las Neurociencias. Juan Pascual-Leone y Robbie Case en Canadá lideraron esta corriente, en la cual también trabajaron y siguen trabajando Mariela Orozco y César Delgado en Cali.

\section{La crítica neo-vygotskiana: del individuo a la sociedad}

Pero la controversia principal no fue entre los piagetianos y las disidencias pospiagetiana y neopiagetiana. Fue la publicación tardía de la crítica de Vygotsky a las ideas iniciales de Piaget.

La crítica de Vygotsky al primer libro de Piaget no se refirió a la existencia del fenómeno del habla egocéntrica que ocurre cuando varios niños juegan juntos, pero cada uno su juego. Se dirigió certeramente a la interpretación del lenguaje egocéntrico infantil como estadio previo a la comunicación lingüística con niños y adultos. Piaget solo conoce estas críticas en los 60, en las traducciones al inglés de Harvard y el MIT. Las acepta parcialmente, pero señala con razón que su obra había continuado 20 años más desde la muerte de Vygotsky. Continuaría otros 20 años más antes de su muerte.

Pero esa pretendida descalificación de Piaget por Vygotsky se extendió ampliamente por razones ideológicas. Se tomó a Piaget como ejemplo de la ideología individualista burguesa, y a Vygotsky como el campeón de la sociedad y el socialismo.

Sin necesidad de descalificaciones ideológicas, Vygotsky hizo aportes teóricos suficientes para darle la vigencia que hoy tiene. La ley de los dos niveles para las funciones superiores del pensamiento, la centralidad del lenguaje y otras mediaciones instrumentales en el aprendizaje y el desarrollo, las relaciones dialécticas entre aprendizaje y desarrollo y sobre todo, la propuesta de la zona de desarrollo próximo o proximal ZDP son contribuciones irreversibles al estudio del desarrollo infantil, del aprendizaje y de la enseñanza. Es verdad que las etapas de Piaget no tienen en cuenta lo que puede hacer el niño con ayuda de adultos o pares más adelantados. Es claro ahora, que con las debidas cautelas, el aprendizaje no tiene que esperar hasta que el niño desarrolle ciertas estructuras mentales que posibiliten funciones superiores de pensamiento, sino que esas estructuras 
mentales y esas funciones superiores se desarrollan con y por el aprendizaje en pequeños grupos, en diálogo con pares un poco más adelantados y con la mediación de los adultos y los instrumentos materiales y simbólicos.

En Colombia, Rosalía Montealegre llegó a Bogotá de la Unión Soviética con estas ideas, Myriam Vega a Cali, y algunos psicólogos chilenos emigrados de la dictadura de Pinochet enseñaron por un tiempo las ideas vygotskianas en la Universidad Incca. Hoy día, después del centenario conjunto del nacimiento de Vygotsky y de Piaget en 1996, no puede hablarse de refutaciones mutuas, sino de complementariedad de puntos de vista que enriquecieron y siguen enriqueciendo la Psicología, la Pedagogía y la Didáctica (ver Castorina, 2007; Castorina y Baquero, R. 2005; Castorina, Ferreiro y Lerner, 1996; Vasco e Isaza de Gil, 2002).

\section{La crítica foucaultiana: de la ciencia al discurso, el saber y el poder}

El Grupo de Historia de las Prácticas Pedagógicas dirigido por Olga Lucía Zuluaga comenzó otro camino que fue desplazando el discurso piagetiano como un discurso más, y la epistemología estructuralista de Bachelard y de Piaget como fijadas en un momento tardío de la formación discursiva moderna, ya a punto de desaparecer.

Cualquier versión de la Psico- logía occidental o de la soviética o de la oriental, si existen, quedan ubicadas en sucesiones o redes de formaciones discursivas adoptadas y abandonadas por los poderes de turno. No hay discursos verdaderos ni falsos, ninguno de ellos tiene fundamentos filosóficos ni científicos privilegiados, ni puede pretender universalidad. Solo hay que esperar y analizar discursos y contradiscursos que surgen y se posicionan como consolidación o resistencia al poder, en una especie de "darwinismo discursivo" en el que todos los discursos modernos van muriendo ante el giro lingüístico, o el giro discursivo que veremos en seguida.

\section{La crítica sociológica: de} los conocimientos a los

\section{saberes y las prácticas}

\section{culturales}

Paralelamente, los sociólogos Bourdieu y Passeron en Francia y Basil Bernstein en Inglaterra proponen otras visiones de los campos intelectuales en los que se dan las discusiones psicológicas, sociológicas, educativas o de otras regiones académicas de fronteras cambiantes.

Esas críticas desde la sociología y la antropología, que se acercan una a otra con Bourdieu y Bernstein, Geerz, Goffman y Garfinkel, Norbert Elias y Anthony Giddens, cuestionan la importancia del discurso psicológico individual y enfatizan los procesos sociales, históricos y culturales. 
En las Matemáticas, y ahora en otras didácticas, el enfoque antropológico de la didáctica de Chevallard, Artigue y Rabardel en Francia, y de Mariana Bosch y Josep Gascón en España, da por terminado el período cognitivo y epistemológico de la didáctica que venía de Piaget y se propone estudiar más bien la emergencia de saberes y discursos a partir de prácticas sociales mediadas por instrumentos materiales y simbólicos, en distintos acercamientos a la conceptualización de las actividades, la acción, las prácticas, los instrumentos y el lenguaje.

\section{La crítica habermasiana: de la razón monológica a la comunicativa}

La psicología cognitiva y la epistemología que el mismo Habermas había promovido en los años 1960-1980 fue abandonada por él con la publicación de los dos tomos de Teoría de la acción comunicativa. Se pasa de la filosofía, la sociología y la psicología monológicas, producidas por un pensador que reflexiona y razona por sí solo, a la razón comunicativa o dialógica, en la que la teoría se va configurando a través de diálogos, acuerdos, consensos y disensos como parte de la acción humana.

Paradójicamente, su valoración de Piaget en la introducción a la Teoría de la acción comunicativa lo hace presente en Alemania, en donde nunca había sido central, y lo rescata del olvido en que había caído en Francia en el tiempo del postestructuralismo de Lacan, Foucault, Derrida, Deleuze, Guattari, Baudrillard, Roland Barthes y Julia Kristeva.

\section{La crítica posmoderna: de lo cognitivo a lo discursivo}

Paralelamente al giro discursivo foucaultiano, el llamado "giro lingüístico" en Gran Bretaña y Estados Unidos provocado por la publicación de las notas de Wittgenstein y consolidado con la publicación del libro con ese título editado por Richard Rorty, llevó a filósofos, psicólogos y educadores a abandonar las teorías estructuralistas y sistémicas y las teorías de etapas o estadios, entre ellas las de Piaget. Para estas corrientes posmodernas, las realidades sociales y personales se crean por los discursos, en particular por las narrativas y las conversaciones cotidianas, y no por ningún tipo de fundamento en la naturaleza humana, de la persona o de la sociedad.

La Psicología individual desaparece en la Psicología social discursiva, y los métodos científicos de las Ciencias Sociales y Humanas se reducen al análisis del discurso en sus distintas vertientes: la de Teun van Dijk en Holanda, Inglaterra y España; la bakhtiniana de Ducrot, 
Todorov, Charaudeau y Maingenau en Francia, y la teoría del posicionamiento de Rom Harré, van Langenhove, Schiffrin, Tannen y Hamilton en la escuela de Georgetown en los Estados Unidos.

La conversión de Rom Harré de ser el más prominente experto en la Psicología cognitiva en Oxford al campeón de la Psicología social discursiva fue paradigmático ${ }^{9}$.

\section{Pervivencia del constructivismo genético piagetiano en el siglo XXI}

A pesar de estas corrientes en las que parece que hubiera desaparecido la piagetiana en el siglo XXI, la veo resurgir por todas partes. En particular, la teoría de modificabilidad cognitiva, basada en las ideas de mediación vygotskianas pero con instrumental cognitivo constructivista de Reuben Feuerstein, ha continuado las ideas de Piaget. En Colombia, el Grupo Cisne y Germán Pilonieta han liderado las ideas de Feuerstein en la Educación.

La didáctica de las Matemáticas como "Mathematics Education" en Estados Unidos: Jeremy Kilpatrick, Leslie Steffe y Ernst von Glasersfeld. La Universidad de Georgia y la revista Journal of Research for Mathematics Education JRME continúa en la línea constructivista en decenas de discípulos de esos maestros, tam-

9 Ver el Handbook of discourse analysis editado por Deborah Schiffrin, Deborah Tannen y Heidi Hamilton. bién con variantes neovygotskianas y dimensiones semiótica potentes, como es el caso de Adalira SáenzLudlowd, Vilma Mora y otros graduados de Georgia.

La didáctica de las Matemáticas en Francia que se inició con Gérard Vergnaud, Guy Brousseau, Régine Douady y muchos colaboradores de la revista Recherches en Didactique des Mathématiques RDM continúa su curso. Ahora se combina y disuelve en vertientes antropológicas y culturales, pero la influencia de Piaget no solo no ha desaparecido, sino que reaparece en formas inesperadas, como en las contribuciones desde la teoría noético-semiótica de Raymond Duval, que en las mismas críticas a Piaget muestra que sin las propuestas constructivistas piagetianas no hubiera podido avanzarse en la teoría didáctica de las Matemáticas.

\section{Conclusión}

Los discursos potentes, serios y coherentes no mueren. Se sumergen parcialmente, resurgen, se mezclan con otros y se prolongan en ellos de maneras variadas, paradójicas y a veces más potentes que los primeros remolinos. Así sucede con los discursos piagetianos en el mundo, y en particular en la Educación colombiana.

Es verdad que los tres estadios piagetianos del desarrollo cognitivo con sus propuestas de edades y estructuras mentales universales quedaron atrás, y que muchos expe- 
rimentos con técnicas más finas han mostrado que en otras circunstancias los niños y niñas sí pueden lograr a edades mucho más tempranas lo que Piaget pensaba que no podían hacer todavía. Pero sin las ideas de Piaget no hubiera sido posible ni siquiera diseñar esos experimentos, y en muchos casos el retroceso en el umbral cronológico no solo no refuta la propuesta conceptual de base, sino que la confirma.

Ya el discurso psicológico piagetiano que tomaba de las matemáticas al estilo del grupo Bourbaki la primacía de las estructuras de grupo, las estructuras de orden y las estructuras topológicas sobre los desempeños concretos de los niños y niñas quedó también atrás. Pero la propuesta de tratar de describir los cambios en las conductas típicas por medio de modelos sistémicos y teorías rigurosas sigue siendo un propósito científico general.

También, quedó atrás el paralelismo entre la ontogénesis y la filogénesis que propuso inicialmente Piaget, pero sigue siendo muy fecundo el poder heurístico para la investigación de los paralelos entre la psicógénesis y la sociogénesis como los propuso Piaget en una obra póstuma completada por Rolando García ${ }^{10}$.

Habría que analizar en detalle todas las ideas piagetianas que se han incorporado al saber tácito de los maestros y maestras colombianos. Por ejemplo, se ha vuelto "natural" reconocer que los niños y niñas no piensan más mal o más débilmente que los adultos, sino que piensan muy bien y muy creativamente, aunque de manera diferente a nosotros y que los niños y niñas desde antes de la educación formal empiezan a construir sus propios modelos mentales y sus teorías personales.

Se ha vuelto obvio para muchos docentes que es necesario ir más allá de los pretests para el establecimiento de "conductas de entrada" a la indagación de los saberes previos de sus estudiantes. No se va a encontrar ningún maestro o maestra que no aprecie la diferencia entre aprender definiciones de memoria y construir conceptos; que no valore el error como fuente de aprendizaje y que no reconozca la larga y difícil conformación de pensamiento formal en los adolescentes y jóvenes.

También se ha extendido la comprensión de la evaluación formativa como diagnóstico de progreso en una construcción conceptual compleja y tortuosa, y como oportunidad de nuevos aprendizajes.

En particular, en la didáctica de las Matemáticas, la enseñanza de la aritmética elemental ha cambiado muchísimo con la teoría piagetiana de la construcción de los números naturales sintetizada en el libro Génesis del número en el niño de Piaget y Szeminska (1941/1975) y en 
el capítulo 1 del primer volumen de la Introducción a la epistemología genética (1950/1978).

Esta teoría se ha propagado en todo el mundo a través de las propuestas de Rochel Gelman y Charlese Randy ("Randy") Gallistel desde 1978 y de Karen Fuson; en Colombia, también por la influencia de Constance Kamii en los años 80 y 90 en de los cursos de capacitación y en los autores de textos escolares.

La Geometría se ha recuperado y transformado en algunos colegios con las ideas de Piaget, Inhelder y Szeminska en La geometría espontánea del niño (1948), y en el libro de Piaget e Inhelder La representación del espacio en el niño, publicado en el mismo año, y en el capítulo 2 del primer volumen de la Introducción a la epistemología genética (1950/1978).

Esta teoría fue propagada mundialmente a través del libro de Holloway (1967/1969) Introducción a la concepción de la geometría en el niño, en la traducción al castellano de Paidós en 1969, libro reimpreso muchísimas veces. Entra también esta teoría a la enseñanza de la geometría a través del discípulo de Piaget, Seymour Papert, como teoría de base para el desarrollo del lenguaje computacional LOGO, y fue propuesta en su exitoso libro de 1980 Mindstorms: Children, computers, and powerful ideas (New York: Basic Books). Este libro fue traducido al castellano como Desafío a la mente. Computadoras y educación en Bue- nos Aires: Galápago en 1981, con varias ediciones posteriores.

Como lo apunté arriba, sin estos aportes de Piaget no habría sido posible el avance sustancial que hace Raymond Duval con su teoría noético-semiótica de los registros de representación para la enseñanza de la Geometría (Duval, 1995).

El libro de Ed Labinowicz (1979/1980) Introducción a Piaget, traducido al castellano inmediatamente por el Fondo Educativo Interamericano, fue influyente en licenciaturas en Educación con énfasis en Matemáticas o en enseñanza de las Ciencias y en multitud de cursos de formación continuada, así como en especializaciones y maestrías.

Como lo dijo ya hace años el editor de la cuarta edición del Handbook of child psychology, Paul Mussen (1983), en este sentido "ahora todos somos piagetianos", pero ya no nos damos cuenta.

En el estilo oratorio propio de tan solemne ocasión, en mi intervención en México para el centenario del nacimiento de Piaget en 1996 amplié esta idea así:

Aun los que prefieren distanciarse de Piaget para proponer sus propias teorías del desarrollo conceptual no tienen más remedio que [p. 198] aceptar que esas ideas no podrían haberse concretado sino en la discusión a favor o en contra de Piaget, y que no habrían podido sobreaguar sino en la estela dejada por el tormentoso paso de la nave piagetiana por los mares del 
análisis experimental de la conducta, vapuleada por sus olas durante más de cincuenta años antes de ganar el reconocimiento que se merecía. (Vasco, 1998, pp. 197-198).

Algo semejante podemos decir ahora con otras metáforas acerca de la influencia de las ideas piagetianas en la Educación colombiana. Las propuestas piagetianas de los años 70 y 80 del siglo pasado rompieron la hegemonía del análisis experimental de la conducta en la Educación e inclinaron la balanza a favor de la Ciencia cognitiva.

Las tesis e hipótesis piagetianas cimentaron los edificios actuales de la didáctica de las Matemáticas y de la didáctica de las Ciencias Naturales como disciplinas científicas, así los pisos superiores actuales de esos edificios apenas en construcción muestren ahora tal variedad de diseños y estilos que no dejan ver los rastros arqueológicos que revelarían claramente cómo y por qué se trazaron así las excavaciones y se construyeron en esa forma los cimientos.

\section{Referencias}

Bang, V., Gréco, P., Grize, J. B., y Piaget, J. (1971). La epistemología del espacio. [Trad. castellana de Jorge A. Sirolli]. Buenos Aires: Ateneo. [Obra original publicada en francés en 1964, Paris: PUF, como n. 18 de los Études d'Épistémologie Génétique].

Castorina, J. A. (2007). Entrevista a José Antonio Castorina. En Revista 12(ntes). Papel y tinta para el día a día en la escuela, n. 17. Recuperado en septiembre de 2007, pp. 10-13, de http://www.12ntes.com/ revista/numero17.pdf.

Castorina, J. A. y Baquero, R. (2005). Dialéctica y psicología del desarroIlo. El pensamiento de Piaget y Vigotsky. Buenos Aires: Amorrortu.

Castorina, J. A., Ferreiro, E. y Lerner, D. (1996). Piaget-Vigotsky. Contribuciones para replantear el debate. Buenos Aires: Paidós.

Flavell, J. H. (1963). Developmental Psychology of Jean Piaget. Princeton, NJ: D. Van Nostrand.

Fuson, K. C. (1988). Children's counting and concepts of number. New York: Springer-Verlag.

Fuson, K. C., \& Carpenter, T. P. (Eds.) (1990). Learning and teaching place value and multidigit addition and subtraction. Madison: Wisconsin Center for Education Research.

Holloway, G. E. T . (1969). Concepción de la Geometría en el niño según Piaget. Buenos Aires: Paidós. (Obra original publicada en ingles en 1967 en Londres por Routledge and Kegan Paul y la National Froebel Foundation). 
Labinowicz, E. (1980). Introducción a Piaget. Pensamiento, enseñanza, aprendizaje. México, DF: Fondo Educativo Interamericano. (Obra original editada en inglés en 1979 con el título The Piaget Primer. Thinking, learning, teaching. New York, etc.: Addison-Wesley).

Mussen, P. H. (Ed.). (1983). Handbook of child psychology (vol. 3: Cognitive development. 4th ed.). New York: John Wiley.

Papert, S. (1981). Desafío a la mente. Computadoras y educación. Buenos Aires: Galápago. (Obra original publicada en inglés en 1980 con el título Mindstorms: Children, computers, and powerful ideas. New York: Basic Books).

Piaget, J. (1978). Introducción a la Epistemología genética. I. El pensamiento matemático [2a. ed.]. Buenos Aires: Paidós. (Obra original publicada en francés en 1950 en Paris: PUF).

Piaget, J. y García, R. (1982). Psicogénesis e historia de la Ciencia. Mexico: Siglo XXI.

Piaget, J. et Inhelder, B. (1948). La représentation de l'espace chez lénfant. Paris: PUF.

Piaget, J., Inhelder, B, et Szeminska, A. (1948). La géométrie spontanée de l'enfant. Paris: PUF.

Piaget, J. y Szeminska, A. (1975). Génesis del número en el niño. ( $5^{a}$ ed.). Buenos Aires: Guadalupe. Obra original publicada en 1941 en Neuchâtel: Delachaux et Niestlé. La primera edición en castellano fue en 1967.
Vasco, C. E. e Isaza de Gil, G. (2002). Piaget y Vygotsky: convergencias y divergencias. En Revista Educación y Pedagogía, 14(33). 223-239.

Vasco, C. E. (1998). La enseñanza de la Física y de las Matemáticas desde la epistemología piagetiana. En: J. A. Castorina, et al., Piaget en la Educación: Debate en torno de sus aportaciones (pp. 195-211). México: Paidós/Universidad Nacional Autónoma de México.

Vasco, C. E. (1995). History of mathematics as a tool for teaching mathematics for understanding. In D. N. Perkins, J. L. Schwartz, M. M. West, \& M. S. Wiske (Eds.), Software goes to school. Teaching for understanding with new technologies (pp. 54-69). New York-Oxford: Oxford University Press.

\section{Apéndice l: Algunos}

\section{artículos del autor sobre} Piaget 1980-2000

(1980). Psicología y Epistemología genéticas. En: G. Aldana de Conde y M. T. González (Eds.). La Psicología, ¿Ciencia Social? (pp. 65-88). Bogotá: Universidad Javeriana.

(1981). Piaget y la enseñanza de la Matemática. En Notas de Matemática, n. 12, 1-18. (En colaboración con Teresa León Pereira). 
(1983). Concepto de la psique en el estructuralismo y en la Epistemología y la Psicología genética. En Encuentros (CAFAM, Bogotá), n. 1, 13-21.

(1984a). Isomorfismos y niveles de resolución en la construcción de modelos. En ICFES: Simposio: ¿Es Posible una Psicología Unificada? (pp. 11-38). [Serie Memorias de Eventos Científicos Colombianos, n. 22]. Bogotá: ICFES/Guadalupe.

(1984b). Invitación a la lectura de Jean Piaget. En Infancia. Bogotá: 1(1), 18-19.

(1989). Dos nuevos grupos piagetianos en la lógica elemental. En Revista de la Academia Colombiana de Ciencias Exactas, Físicas y Naturales. 17(64), 29-39.

(1999). Críticas contemporáneas al constructivismo: El constructivismo, ¿misión imposible? En: C. E. Vasco (Ed.), Constructivismo en el aula: ¿llusiones o realidades? (pp. 15-33). Santafé de Bogotá: CEJA.

(2006). Didáctica de las Matemáticas. Artículos selectos. Bogotá: Universidad Pedagógica Nacional.

\section{Apéndice Il: Algunas publicaciones del grupo de Epistemología de la Facultad de Ciencias de la Universidad Javeriana}

Vasco, C. E. (Ed.). (1999). Constructivismo en el aula: ¿llusiones o realidades? Bogotá: CEJA.

Vasco, C. E. (Ed.). (2000). Construyendo una propuesta pedagógica práctico-téorica: Crónicas de una experiencia. Bogotá: Centro Editorial Javeriano CEJA. (Con otros autores: Vasco, C. E.; Barrera de Aragón, M.; Quijano de Castellanos, M. V.; Suárez Gómez, A.; Amat, G. D.).

Vasco, C. E. e Isaza de Gil, G. (2002). Piaget y Vygotsky: convergencias y divergencias. En Revista Educación y Pedagogía. 14(33), 223-239.

Vasco, C. E. (Ed.). (2003). Posmodernidad, ciencias y educación. Bogotá: Centro Editorial Javeriano CEJA.

Vasco, C. E. (Ed.). (2004). De la teoría a la práctica en la formación de maestros en ciencias y matemáticas en Colombia. Bogotá: Editorial Pontificia Universidad Javeriana. (Editor y co-autor con Barrera de Aragón, María; Becerra Nivia, María Haydée; Suárez Gómez, Alfonso; Perdomo de Parra, Nubia). 